\title{
Bargeld in privaten Geldsystemen
}

\section{Einleitung}

Das Verschwinden des Bargelds kennzeichnet einen tiefgreifenden Wandel des Geldes in der fortschreitenden Moderne, in dem Geld sich immer weiter ausdifferenziert und privatisiert. Längst liegt der Großteil des Geldes in unbarer Form vor, als von den privaten Geschäftsbanken erzeugtes Giralgeld. Der bargeldlose Zahlungsverkehr wird durch Zahlungsinstrumente wie Überweisung, Lastschrift, Bank- oder Kreditkarten abgewickelt und ersetzt zunehmend die bisherigen monetären Transaktionen mit dem staatlich legitimierten Zahlungsmittel des Bargeldes. Neben den Banken bieten inzwischen zahlreiche private Zahlungsdienstleister wie paypal, giropay, paysafecard oder Western Union Zahlungsmittel und Zahlungsverfahren an. Hinzu kommen diverse außerhalb des offiziellen Zentralbankgeldsystems geschöpfte Kryptowährungen wie Bitcoins, Ether oder Dogecoins. „Das“ Geld wird damit vielfältiger, virtueller, privatisierter und grenzüberschreitender. Es beginnt den nationalstaatlichen Rahmen zu verlassen, in den es die letzten 150 Jahre eingebunden war, und löst sich dabei auch immer stärker von seiner bisherigen Gestalt als Banknote und Münze. Diese Entwicklung wird begleitet von einer lebhaften Diskussion, in der eine Seite die Abschaffung des Bargeldes fordert (um Kriminalität zu unterbinden oder Konjunkturpolitik mit Negativverzinsung zu ermöglichen; vgl. Rogoff 2016), während die andere Seite dies vehement ablehnt (etwa aus Gründen der informationellen Selbstbestimmung; vgl. Häring 2016). Diese Debatte steht hier jedoch nicht im Fokus. Vielmehr soll der Frage nachgegangen werden, ob das Medium „Bargeld“ jenseits des offiziellen Geldsystems eine Zukunft hat. Betrachtet werden hierbei nicht-kommerziell ausgerichtete private Geldsysteme (sog. „Komplementärwährungen“), die ebenfalls einen beachtenswerten Aspekt der Ausdifferenzierung und Privatisierung des Geldes der letzten Jahrzehnte darstellen.

\section{Komplementäre Währungen weltweit}

Komplementärwährungen sind Geldsurrogate, die parallel (nicht alternativ!) zur vorherrschenden Nationalwährung existieren ${ }^{1}$. Sie beruhen auf der Vereinbarung einer

\footnotetext{
${ }^{1}$ Zur Einführung in die Thematik siehe Gründler 2005; Kennedy/Lietaer 2004; North 2010; Plettenbacher 2008. Detailliertere Beschreibungen von Komplementärwährungen finden sich etwa unter http://complementarycurrency.org/ oder http://monneta.org/. Wissenschaftliche Forschung zu dem Thema
} 
Gemeinschaft, etwas (Gutscheine, virtuelle Kredite, Zeiteinheiten, etc.) neben dem offiziellen Geld als Zahlungsmittel zu akzeptieren (Seyfang und Pearson 2000, S. 57). Seit den 1990er Jahren sind weltweit tausende solcher Systeme entstanden (Kennedy und Lietaer 2004, S. 73) ${ }^{2}$. Trotz großer Unterschiede lassen sich alle letztlich als Reaktion auf aktuelle wirtschaftliche und soziale Entwicklungen verstehen, seien dies die Erosion traditioneller sozialer Netzwerke, steigende Arbeitslosigkeit, die Krise des regionalen Einzelhandels oder eine, entfesselte Finanzwirtschaft'. Viele dieser Problemlagen werden von bestimmten gesellschaftlichen Gruppen wahrgenommen und auf das herrschende Geldsystem zurückgeführt. Daraus entwächst die Motivation eigene Gelder zu emittieren, die bestimmte Ungleichgewichte kompensieren sollen ${ }^{3}$. Je nach wahrgenommener Problemlage entstehen unterschiedliche Varianten: Regionalgelder wollen die regionale Wirtschaft stärken, Tauschringe praktizieren Vergemeinschaftung, Zeitbanken fördern Ehrenamt und soziales Engagement, Seniorengenossenschaften versuchen die Versorgungslage von Rentnern zu verbessern und die Gold- und Silbermünzen privater Organisationen in den USA zielen auf Sicherheit und Unabhängigkeit (für eine Klassifikation siehe etwa Blanc 2011). All dies sind offenbar Versuche, unterschiedliche Geldarten $\mathrm{zu}$ konstruieren, die jeweils eigene Anreizstrukturen für bestimmte Verhaltensmuster beinhalten (Lietaer et al. 2008). Die maßgebliche Grundidee lautet: Geld ist ein grundsätzlich positives Instrument, das momentan nur wegen bestimmter „Konstruktionsfehler“ oder in bestimmten Ausprägungen negativ

wird u.a. orchestriert von der ,Research Association on Monetary Innovation and Community and Complementary Currency Systems“ (http://ramics.criterical.net/) und oftmals publiziert im Online-Journal „International Journal of Community Currency Research“ (https://ijccr.net/). Eine umfangreiche Datenbank mit einschlägiger Literatur bietet die von Rolf Schröder herausgegebene „Bibliography of Community Currency Research“ (http://cc-literature.org/).

2 Ein relativ aktueller Forschungsüberblick listet weltweit 3418 Komplementärwährungen auf (Seyfang/Longhurst 2013).

3 Die grundsätzliche, wenn auch in ihren Details verschiedene idealistische Intention dieser privaten Geldsysteme ermöglicht eine (wenn auch nicht absolut trennscharfe) Abgrenzung von kommerziell ausgerichteten privaten Geldsystemen. Unter letztere fallen etwa: Barterorganisationen, die teilnehmenden Unternehmen einen multilateralen Tausch über verschiedene Verrechnungssysteme ermöglichen; TokenSysteme wie die (inzwischen eingestellten) ,MicrosoftPoints' oder ,Facebook-Credits', die bei den beiden Unternehmen als interne Währung für den Kauf verschiedener Produkte und Dienstleistungen verwendet werden können; Bonusprogramme (Miles\&More, Happy Digits etc.), bei denen eigene, oft nur begrenzt einsetzbare Währungen (Loyality Tokens) der Kundenbindung dienen; Gutscheinsysteme wie die von der ,Schweizer Reisekasse' herausgegebenen ,Reka-Checks', mit denen in Verkehrs- und Tourismusbetrieben bezahlt werden kann, oder die in Frankreich als Lohnnebenleistung gewährten ,Tickets Restaurant', die in vielen Restaurants und Lebensmittelläden wie Geld verwendet werden können. Man könnte noch eine Vielzahl weiterer derartiger Geldsubstitute nennen, der entscheidende Punkt ist: Sie alle dienen kommerziellen Interessen, indem sie etwa Unternehmen zusätzliche Liquidität für ihren Austausch zur Verfügung stellen, steuer- und sozialabgabenfreie Gehaltszulagen ermöglichen, Konsumenten durch Verwendungseinschränkung des Geldsurrogats an Produkte und Unternehmen binden usw. Entsprechend sind diese kommerziellen Währungen zumeist von Unternehmen initiiert, während die ideellen Komplementärwährungen von zivilgesellschaftlichen Akteuren herausgegeben und deswegen auch als „grassroot currencies“ (Seyfang/Longhurst 2012) bezeichnet werden. 
wirkt. Man kann ihm mittels unterschiedlicher Konstruktionsarten verschiedene Ziele „einprogrammieren“ und es damit veranlassen, ,sozialen Nutzen [zu] stiften“ (Kennedy 2005, S. 20). Dementsprechend wollen Komplementärwährungen, Werkzeuge' zur Lösung aller möglichen Probleme sein. In ihren theoretischen Grundlagen und praktischen Intentionen unterscheiden sich die Systeme jedoch stark, was daran liegt, dass sich unterschiedliche soziale Bewegungen diese Grundidee vor dem Hintergrund ihrer je eigenen ideologischen Konzeption zu Eigen machen (vgl. dazu Degens 2013; Thiel 2011).

Betrachten wir im Folgenden einige dieser Komplementärwährungen, und zwar entsprechend der hier verfolgten Fragestellung - solche, die mit physischem Geld operieren. Ausgeklammert werden damit beispielsweise Tauschringe oder Zeitbanken, die mit gegenseitigen Krediten arbeiten und diese als virtuelle Währungen in Form von Kontoständen verwalten (ausführlich dazu Hoeben 2003; Meier 2001; Seyfang 2002).

Die meisten der bargeldbasierten Komplementärwährungen sind sog. regionale Währungssysteme („Regionalgelder“). Kerngedanke ist, das jeweilige Geld durch seine räumlich beschränkte Gültigkeit an die Region zu binden, um so Kapitalabfluss zu verhindern und die regionale Ökonomie zu stärken. Die erste dieser modernen Regionalwährungen wurde 1991 von Paul Glover in der kleinen Universitätsstadt Ithaca als Mittel der ökonomischen Selbsthilfe entwickelt. Er konzipierte sein „Ithaca Hours“ genanntes Geld als ungedeckte Papierwährung, deren Werteinheit in Zeiteinheiten (von einer Viertelstunde bis zu vier Stunden, wobei eine Stunde mit 10 US\$ gleichgesetzt wurde) ausgedrückt wurde. Auf dem Höhepunkt ihrer Popularität Mitte der 1990er Jahre verwendeten Tausende in Ithaca und Umgebung die Ithaca Hours für ihre Ein- und Verkäufe (Jacobs et al. 2004). Die Idee verbreitete sich rasch und allerorts entstanden Ableger, beispielsweise die UkiahHours, die PeakHours oder die RiverHours. Allerdings ist die Kontrolle von Werthaltigkeit, Geldumlauf und Geldschöpfung bei solchen ungedeckten Papierwährungssystemen schwierig, weshalb ab Ende der 1990er Jahre zunehmend Regionalwährungen entstanden, die durch die jeweilige Nationalwährung gedeckt waren. In solchen Systemen tauschen die Teilnehmer ihr offizielles Geld in die jeweilige Regionalwährung um, die in begrenztem Maße auch wieder zurücktauschbar ist. In den USA entstanden u.a. die BerkShares (Region Massachusetts; seit 2006), in Kanada der Calgary Dollar (seit 1996), die Salt Spring Dollars (seit 2001), die

\footnotetext{
${ }^{4}$ Der Wert dieses Geldes basiert also allein auf der Vereinbarung der Teilnehmer, es als Geld zu akzeptieren. Während bei Tauschringen die (virtuelle) Währung im Tauschvorgang oder bei den eurogedeckten Regionalgeldern vorwiegend durch Eintausch von National- in Regionalwährung entsteht, kommen die Ithaca Hours durch Schenkungen an Teilnehmer (jeder erhält jährlich das Äquivalent von 20\$) oder regionale Nonprofit-Organisationen, als Vergütung für die Kosten der Trägerorganisation oder als zinslose Kredite in den Umlauf.
} 
Kawartha Loons (Peterborough, Ontario; seit 2013) und die Cochrane Dollars (seit 2017), in Großbritannien etwa das Totnes Pound (seit 2006), das Bristol Pound (seit 2012) und das Exeter Pound (seit 2015). Auch außerhalb des englischsprachigen Raums entwickelten sich einige regionale Währungen, beispielsweise in Thailand 1998 der „Bia Kud Chum“, in Mexiko 1996 der „Tlaloc“ und in Argentinien Mitte der 1990er Jahre der „Credito“.

\section{Regionalgelder in Deutschland}

In Deutschland entstand die Idee eines nur regional gültigen und dabei gemeinnützigen Geldes in einem ganz speziellen Kontext. Die ideengeschichtlichen Wurzeln der deutschen Regionalgelder liegen in Freiwirtschaft, Anthroposophie und den neuen sozialen Bewegungen der 1960er Jahre (ausführlich siehe Thiel 2011). Silvio Gesells (1862-1930) Konzept des Freigeldes sieht den maßgeblichen Fehler des Geldsystems in der Wertaufbewahrungsfunktion des Geldes und fordert ein negativ verzinstes Geld. Um Gesell herum entstand eine soziale Bewegung, die in den 1930er Jahren einige praktische Experimente mit Freigeld (etwa in Wörgl) durchführte, die jedoch rasch von Notenbanken verboten wurden. In den 1960er Jahren verbanden sich Teile der Freigeldbewegung mit der Anthroposophie, einer von Rudolf Steiner (1861-1925) gegründeten esoterischen Bewegung, die ein ähnliches Geldkonzept hatte, sowie den Neuen Sozialen Bewegungen mit ihren ökosozialen Intentionen. So entstand ein beachtliches, wenn auch nur lose verbundenes Netzwerk. Anfang der 1980er Jahre wurden mit den Tauschringen erneut praktische Geldexperimente durchgeführt. Allerdings blieben die Tauschringe eher klein und milieuspezifisch, weshalb man um die Jahrtausendwende nach neuen Möglichkeiten zur Durchsetzung einer Alternativ-Ökonomie suchte. Globalisierungskritische Tendenzen sowie die (v.a. in Deutschland) durchaus kritisch beäugte Einführung des Euros beförderten sicher die Entstehung der Idee einer regionalen Währung. Der Startschuss für die deutschen Regionalgelder fiel (nach zwei eher unbeachtet gebliebenen Initiativen 1998 bei Erfurt und 2001 in Bremen) im bayerischen Prien am Chiemsee im Jahr 2003. Der Wirtschaftslehrer Christian Gelleri initiierte, zunächst als Schülerprojekt an der dortigen Waldorfschule, den „Chiemgauer“ - das bislang erfolgreichste Regionalgeld und Auslöser eines regelrechten Booms. In der Folge entstanden in den 2000er Jahren im Bundesgebiet zahlreiche Regionalgelder (zeitweise über 40 aktive Initiativen) mit Namen wie Ammerlechtaler, Bürgerblüte, Dreyecker, Elbtaler, Havelblüte, KannWas, Landmark, Nahgold, Roland, Sterntaler, TauberFranken oder Zschopautaler. Bei ihnen allen handelt es sich um regional gültige und von mehreren Teilnehmern akzeptierte komplementäre Geldarten privater 
Emittenten in Form von Bargeld $^{5}$, die mit einer negativen Verzinsung (oder zumindest Zinslosigkeit) versehen sind und gemeinnützige Ziele verfolgen, wobei sich in den Details (Konstruktionsweise, Zielsetzungen, Teilnehmerkreis) viele Unterschiede finden.

Praktisch funktionieren die Regionalgelder wie folgt (hier am Beispiel des Chiemgauers illustriert): Ein Verbraucher tauscht in einer Ausgabestelle (teilnehmende Geschäfte oder Banken) Euros gegen den gleichen Betrag in Chiemgauer-Scheinen ein. Damit kann er in den teilnehmenden Geschäften einkaufen. Die Geschäftsleute wiederum können den Chiemgauer beim Verein in Euro zurücktauschen, der dabei fällige Abschlag von $5 \%$ kommt teilweise Vereinen und sozialen Einrichtungen in der Region zugute. Zudem verliert der Chiemgauer durch seine negative Verzinsung an Wert, d.h. jedes Quartal muss der Schein mittels einer Klebemarke, die $2 \%$ des Nennwertes kostet, ,erneuert' werden. Auf den ersten Blick erscheint ein solches Geldsystem ökonomisch irrational. Bei genauerem Hinsehen zeigt sich jedoch, dass die beteiligten Gruppen jeweils eigene Vorteile daraus ziehen (ausführlich siehe Thiel 2011): Die Initiatoren des Regionalgeldsystems, die sich (insgeheim) als Protestbewegung sehen, wollen damit als „moralische Unternehmer“ (Becker 1973, S. 133) die Gesellschaft von einem anderen und besseren Geldsystem überzeugen. Für die teilnehmenden Geschäftsleute ist das Regionalgeld ein Werbe- und Marketinginstrument, das die Bekanntheit des Geschäfts steigert, einen Konkurrenzvorteil darstellt und ein positives Image verleiht. Die teilnehmenden Verbraucher wiederum können mit jedem Umtausch eine kleine Spende an ,ihren' Verein bewirken. Wichtiger noch ist für sie die moralische Komponente des Einkaufens mit Regionalgeld: Aufgrund dessen Einschränkungen und symbolischer Aufladung nutzen Verbraucher es als Selbstbindungsmechanismus (man kann nur in den teilnehmenden regionalen - und damit als „gut“ erachteten - Geschäften einkaufen), der gleichzeitig einer gewissen Selbstemblematisierung von bestimmten regional, ökologisch oder sozial orientierten Einstellungen dient. Dies ermöglicht es, im Rahmen der alltäglichen Einkaufsroutinen soziales Engagement (in Form von Spenden) und moralisch korrekten Konsum quasi automatisch abzuwickeln.

\section{Aktueller Stand und zukünftige Entwicklung}

Nach der Boom-Phase der 2000er Jahre ist die Entwicklung von bargeldbasierten Komplementärwährungen, v.a. der deutschen Regionalgelder, inzwischen rückläufig. Viele Regionalgeld-Initiativen sind mittlerweile gescheitert. Die Gründe hierfür sind vielfältig: Man

\footnotetext{
${ }^{5}$ Zumeist verwenden die Regionalgelder (häufig aufwendig gestaltete) Papierscheine, einige wenige auch Münzen (vgl. Sademach 2010).
} 
konnte nicht annähernd die Wirkungen erzielen, die man sich erhofft hatte ${ }^{6}$. Es gelang - trotz mitunter gelungenem und aufwendigem Marketing - nicht, eine ausreichende Akzeptanz und Verbreitung der neuen Geldform zu erreichen (ein Problem, das auch wirtschaftliche „Big Player“ kennen - man denke beispielsweise an das Scheitern der Smartphone-Geldbörse MyWallet der Telekom oder der PayCard von Telekom und Deutscher Bahn). Und nicht zuletzt zeigte sich, dass Regionalgeld ein höchst kosten- und arbeitsintensives Projekt ist, das zwar anfangs, aber nicht dauerhaft ehrenamtlich bewerkstelligt werden kann ${ }^{7}$. Heute gilt das Währungsmodell „Regionalgeld“ in Deutschland als gescheitert und es entstehen kaum mehr neue Projekte. Dies mag daran liegen, dass sich die wirtschaftliche Gesamtlage in Deutschland geändert hat. Zu Anfang des Jahrtausends befand sich die deutsche Wirtschaft in einer Stagnationsphase, der Konsum schwächelte und die Krise des Einzelhandels sowie die Bedrohung der regionalen Wirtschaft durch die Globalisierung wurden häufig thematisiert ${ }^{8}$. Hinzu kommt, dass sich die den Regionalgeldern zugrunde liegende soziale Bewegung inzwischen fast aufgelöst hat. Der Kern dieser Bewegung war der Praxisbezug - man wollte nicht mehr nur über ein „besseres Geld“ theoretisieren, man wollte ein solches in der Praxis umsetzen. Nachdem sich dies deutlich schwieriger gestaltete, als ursprünglich gedacht, verlor die Bewegung viel von ihrer Schubkraft. Inzwischen betreten neue Akteure die Bühne und betreiben (in verschiedenen Ländern) die Weiterentwicklung der Komplementärwährungen. Sie verstehen sich häufig als ,social entrepreneurs“ und knüpfen zwar an die ursprünglichen Ideen und Intentionen an, entideologisieren diese aber deutlich bzw. streben eine höhere Rentabilität und Profitabilität an. Ein Aspekt davon ist die Abwendung vom Bargeld.

Diese Komplementärwährungen der neueren Generation (siehe dazu Blanc 2011; Fare und Ahmed 2017) nutzen vorzugsweise elektronische Zahlverfahren. Um die technische Infrastruktur und die nötige Organisationsstruktur $\mathrm{zu}$ finanzieren, gehen sie vielfältige Kooperationen mit lokalen Regierungen und Verwaltungen, mit Unternehmen und Non-

${ }^{6}$ Beim Chiemgauer, der bei weitem erfolgreichsten Regionalwährung, werden zwar mittlerweile von über 3.000 teilnehmenden Verbrauchern bei fast 600 Unternehmen über 7 Millionen Euro Umsatz generiert und mehr als 60.000 Euro Begünstigungen an Vereine und Projekte ausgeschüttet (http://www.chiemgauer.info/fileadmin/user_upload/Dateien_Verein/Chiemgauer-Statistik.pdf [01.09.2017]). In ökonomischer Hinsicht ist dies jedoch vernachlässigbar gering (ca. 0,01 Prozent des Bruttoinlandsproduktes der Region). Noch ernüchternder ist die Bilanz, wenn man die anderen Regionalwährungen betrachtet. Zumeist sind diese sehr klein, wenige tausend Euro werden von einigen Überzeugungstätern in der immer gleichen Handvoll Geschäfte ausgegeben.

${ }^{7}$ Der Chiemgauer hat es als einzige Initiative geschafft, eine tragfähige (aber immer noch großteils ehrenamtlich basierte) Organisationsstruktur aufzubauen, was auch daran liegt, dass er sein „Geschäftsmodell“ mittels der Sozialgenossenschaft Regios eG auf Mikrofinanzierungen ausgeweitet hat.

${ }^{8}$ Nicht ohne Grund entstehen viele neue Komplementärwährungen in ökonomisch schwierigen Ländern wie Spanien, Portugal oder Italien. Möglicherweise entsteht gerade in Krisenzeiten ein Bedarf an Alternativen zum offiziellen Geld. So hat etwa der „Credito“ während der Währungskrise in Argentinien 1998-2002 kurzzeitig extrem an Bedeutung und Verbreitung gewonnen. 
Profit-Organisationen sowie mit nationalen und internationalen Förderprogrammen ein ${ }^{9}$. Allerdings scheint gerade die Kooperation mit Politik, Wirtschaft und Non-Profit-Sektor neue Probleme für die Komplementärwährungen aufzuwerfen, beispielsweise die teils unterschiedlichen Zielsetzungen und Anforderungen sowie die potentielle Unzuverlässigkeit der Förderung ihrer „stakeholder“ (Blanc und Fare 2013). Hinzu kommt, dass sich die technischen Umsetzungen als mitunter schwierig und kostspielig erwiesen haben (Fare und Ahmed 2017, S. 855). Dies führt uns zu den Vor- und Nachteilen von Bargeld in Komplementärwährungssystemen.

\section{Bargeld in Komplementärwährungssystemen - eine Diskussion}

Beginnen wir mit den häufig genannten Nachteilen von Bargeld in Komplementärwährungssystemen: Währungssysteme, die mit physischem Geld operieren, stehen vor eine Reihe von Herausforderungen, die Aufwand und Kosten mit sich bringen. Banknoten und/oder Münzen erfordern seitens der herausgebenden „Zentralbank“ eine aufwendige Gestaltung (auch damit diese Wert symbolisieren), einen möglichst fälschungssicheren Druck (Spezialpapier, aufwendige Druckverfahren, Sicherheitsmerkmale), eine umsichtige Kontrolle der Geldmenge sowie vielfältige administrative Aufgaben wie Herausgabe, Umtausch, Lagerung, Transport, ständige Echtheitskontrollen usw. (zu Aspekten des Produktionskontexts von Banknoten siehe Thiel 2013, S. 201-205). Komplementäre Währungen müssen Marketing betreiben, um die Bekanntheit ,ihres ' Geldes zu steigern, und brauchen klare Regelungen, wie dieses zur jeweiligen offiziellen Währung steht (Konvertibilität). Auch Handel und Konsumenten haben diverse Aufwendungen: sie müssen die jeweilige Währung eintauschen (und ggf. zurücktauschen), transportieren, aufbewahren, ggf. verbuchen usw. $^{10}$ Amy Kirschner kommt deswegen in ihrer Fallstudie über die

9 Ein Beispiel für eine solche neuere Komplementärwährung ist der NU-Spaarpas, ein Komplementärwährungssystem in Rotterdam, das eine Mischung aus elektronischer Bankkarte und LoyalityPunkte-Programm darstellte. Für nachhaltiges (Konsum-)Verhalten wie den Einkauf von lokalen, biologischen oder fair gehandelten Produkten oder Müll-Recycling wurden der Karte Punkte gutgeschrieben, die dann in teilnehmenden Geschäften ausgegeben werden konnten. Als Partner und Förderer beteiligten sich lokale und kommunale Regierungen, eine Bank und sogar die Europäische Union. Das System florierte zwischen 2002 und 2003 und hatte zu Spitzenzeiten über 10.000 Teilnehmer und ca. 100 teilnehmende Unternehmen. Als jedoch 2003 die Rotterdam Municipal Authority ihre Unterstützung einstellte, war das System am Ende. Vgl. http://community-currency.info/de/braucht-ubersetzung/nu-spaarpas/ [19.09.2017].

10 Viele dieser Punkte gelten natürlich auch für das offizielle Bargeld. So hat etwa eine (von Mastercard finanzierte) Studie des Research Center for Financial Studies der Steinbeis Hochschule die Kosten für die Bargeldversorgung in Deutschland auf jährlich acht Milliarden Euro taxiert. Der größte Teil entfällt dabei auf den Einzelhandel (Personal, Transport, Lagerung, Versicherung) und die Banken (Schaltermitarbeiter, Geldtransporter und -automaten) (Kleine et al. 2013). Dem muss man allerdings entgegenhalten, dass auch elektronische Zahlverfahren Kosten nach sich ziehen (Infrastruktur usw.). Die tatsächlichen Herstellungsund Verwaltungskosten der Zentralbank sind eher gering: Prägen und Druck belaufen sich auf ca. 72 
Papierwährung „Slices“ des Burlington Currency Projects in Vermont, USA zu dem Schluss: „Paper currencies are expensive and hard to administer“ (Kirschner 2011, S. 54). Auch wenn alle Komplementärwährungen mit der Aufgabe konfrontiert sind, die für das reibungslose Funktionieren ihres Geldsystems notwendige Finanzierung (von Organisation, Infrastruktur, Personal) auf Dauer sicherzustellen (vgl. dazu Schröder 2015), schlagen die mit der Bargeldherstellung verbundenen Fixkosten hier besonders zu Buche (und zwar umso stärker, je geringer die Verbreitung des jeweiligen Geldes). Deshalb operieren viele neuere Komplementärwährungen mit elektronischen Zahlverfahren ${ }^{11}$. Selbst etliche der älteren Regionalwährungen haben inzwischen elektronische Zahlverfahren eingeführt - das Bristol Pound etwa das mobile payment via SMS (Ferreira et al. 2015) und der Chiemgauer die „Regiocard“, mit der Verbraucher per Kartenlesegerät ihr normales Bankkonto belasten können. Die Einführung solcher elektronischer Zahlverfahren findet - neben der Vereinfachung von Transaktion und Verbuchung - wohl auch deswegen statt, weil die jeweilige Komplementärwährung dadurch moderner, zukunftsträchtiger und womöglich auch glaubwürdiger wirkt. Die Digitalisierung eröffnet somit Chancen für die weitere Entwicklung von monetären Alternativen, nicht zuletzt hinsichtlich einer möglichen Vernetzung und Tauschbarkeit der verschiedenen Komplementärwährungen (Schröder 2016). Ist also auch bei den Komplementärwährungen das Bargeld am Verschwinden? Ist das elektronische Geld das bessere, weil einfachere, kostengünstigere und zukunftsträchtigere?

Keineswegs, denn Bargeld hat auch diverse Vorteile. Carl-Ludwig Thiele, Vorstandsmitglied der Deutschen Bundesbank, führt eine ganze Reihe an guten Argumenten für das offizielle Bargeld an, die ebenso für Komplementärwährungen gelten.

„Das Bargeld wird als einfaches, sicheres und schnelles Zahlungsmittel wahrgenommen. Bargeld wird auch als Instrument zur Haushaltsplanung verwendet, denn viele Bürgerinnen und Bürger berichten, dass sie bei der Verwendung von Bargeld einen besseren Überblick über ihre eigenen Ausgaben haben. Bürgerinnen und Bürger schätzen das Bargeld zudem als anonymes Zahlungsmittel, das es ihnen erlaubt, ihre Privatsphäre zu schützen und ihr Recht auf informationelle Selbstbestimmung auszuüben. Bargeld ist schlussendlich auch von technischer Infrastruktur weitgehend unabhängig und kann als gesetzliches Zahlungsmittel immer und überall verwendet werden, insbesondere auch in einem Krisenfall. Zusammenfassend lässt sich sagen, dass das Bargeld als modern, effizient und nutzenstiftend wahrgenommen wird“ (Thiele 2015, S. 6).

Millionen Euro pro Jahr, Recycling- und Overheadkosten (inkl. Transport und Verarbeitung) auf etwa 174 Millionen Euro (Kleine et al. 2013, S. 37).

11 Dafür gibt es inzwischen auch schon einige frei verfügbare software- und webbasierte Systeme, auf die kostenfrei zurückgegriffen werden kann. Beispiele hierfür sind mobile banking software Cyclos oder die Internet-Plattform „Community Exchange System“, die sogar den weltweiten Austausch zwischen verschiedenen virtuellen Komplementärwährungen ermöglicht (https://www.cyclos.org/ und https://www.community-exchange.org/ [08.09.2017]) 
In diesem Zitat klingen einige Punkte an, die in einer kurzen Gegenüberstellung von physischem und virtuellem Geld diskutiert werden sollen. Sie betreffen im Wesentlichen zwei Aspekte eines Währungssystems: den technischen und den kulturellen.

\subsection{Technisch-funktionale Aspekte: Kosten und rechtliche Lage}

Bargeld ist, wie oben angeführt, mit Kosten verbunden. Das bedeutet im Umkehrschluss aber nicht, dass elektronisches Geld kostengünstig ist. Thiele (2015, S. 4) bezweifelt nicht ohne Grund die angebliche Kostenersparnis beim elektronischen Zahlungsverkehr. Erstens erfordert auch dieser eine (in Anschaffung und Wartung) mitunter kostspielige technische Infrastruktur (etwa Point-of-Sale-Terminals). Falls diese nicht vorhanden ist, müssen die Transaktionen aufwendig per Hand gebucht werden - so etwa in dem oben genannten Community Exchange System, bei dem die konkrete Bezahlung auf Papierzetteln dokumentiert und später ins System gebucht wird. Die diesbezüglichen Erfahrungen vieler Regionalwährungen lassen sich auf eine simple Formel bringen: Beim elektronischen Geld sind die Initialkosten hoch, die Betriebskosten niedrig; beim Bargeld verhält es sich genau umgekehrt. Zweitens sind elektronische Systeme anfällig für Datenverlust und -manipulation (Hacker) und werfen datenschutzrechtliche Fragen auf - schließlich werden alle mit dem jeweiligen elektronischen Geld vollzogenen Transaktionen dokumentiert.

Noch wichtiger ist für Komplementärwährungen die rechtliche Lage. Geld ist ein Geschöpf der Rechtsordnung und das Geldsystem „ein staatlich zugelassenes, überwachtes und gestütztes Kooperations- und Kapitalbildungssystem“ (Reifner 2017, Band III, S. 5). Der Staat schützt sein institutionalisiertes Geldmonopol mit strengen währungsrechtlichen Vorgaben (etwa §35 BbankG). Bargeldbasierte Komplementärwährungen müssen deswegen als ausschließlich vereinsinternes und freiwilliges Zahlungsmittel, als „Gutschein“ konzipiert sein, für den ebenfalls zahlreiche Rechtsvorschriften gelten (siehe dazu Hardraht/Godschalk 2004). Allerdings unterliegen auch virtuelle Währungen diversen Rechtsverordnungen (etwa dem Bundesbankgesetz, dem Kreditwesengesetz oder den EU-Richtlinien zu E-Geld und Zahlungsdiensten), denn ,auch beim Giralgeld (Privatgeld) ist der Staat entscheidend“ (Reifner 2017, Band III, S. 5). Diese (aufsichts-)rechtliche Giralgeldregulierung stellt eine große Hürde für Regionalwährungen dar, vor allem wenn eine (teure und aufwendige) EGeld-Lizenz entsprechend des Kreditwesengesetzes (KWG) fällig wird. In Deutschland dulden die Bundesbank, die BaFin und andere staatliche Geldakteure die Komplementärwährungen - ob bargeldbasiert oder virtuell - aufgrund ihrer geringen 
Umsätze. Es ist allerdings davon auszugehen, dass gerade virtuelle Komplementärwährungssysteme zunehmend in den Blick der Aufsichtsbehörden geraten. So hat die EU-Kommission unlängst einen Entwurf ${ }^{12}$ vorgelegt, der vorsieht, virtuellen Währungen geldwäscherechtliche Pflichten aufzuerlegen. In Anlehnung an eine EZB-Studie zu virtuellen Währungen (EZB 2012) werden virtuelle Währungen dabei sehr weit definiert und könnten somit beispielsweise auch digitales Spielgold, Facebook Credits, Nintendo Points oder Vielflieger-Bonusmeilen umfassen. Folglich würden neben den Börsen für Bitcoin und Co. auch Verkäufer von Spielwährung und Ähnlichem unter die neuen Geldwäscheregularien fallen. Momentan schließt der Entwurf komplementäre Währungen dezidiert aus ${ }^{13}$. Dies könnte sich jedoch schnell ändern, wenn Komplementärwährungen - wie schon in Ansätzen erkennbar - zunehmend auf größere Verbreitung, höhere Skalierbarkeit und gegenseitige Konvertibilität setzen (was mit physischem Geld deutlich schwieriger umzusetzen ist als mit elektronischem).

Insgesamt sind also virtuelle Komplementärwährungen gegenüber bargeldbasierten weder zwingend kostengünstiger noch rechtlich unstrittiger.

\subsection{Kulturelle Aspekte: Symbolik und Zahlungsgewohnheiten}

Kaum beachtet, aber von entscheidender Bedeutung, sind die kulturellen Aspekte des Geldes. Geld ist nicht nur abstraktes Tauschmittel und rationales Schlüsselwerkzeug des modernen Wirtschaftslebens; es existiert gleichzeitig außerhalb der Sphäre des Markts und wird in hohem Maße von kulturellen und sozialen Strukturen beeinflusst (Zelitzer 1989). In der Alltagspraxis gewinnt Geld eine multiple Symbolik - je nach Herkunft, Verwendung und jeweiliger Materialität (Carruthers 2002).

Nehmen wir einen Aspekt dieser Materialität: die visuelle Gestaltung des Geldes. Diese ist ein essentieller Bestandteil der für das Funktionieren eines Geldsystems so wichtigen „vertrauensbildenden Maßnahmen“ (Gabriel 2002, S. 36). Ein Blick in die Geschichte und Gegenwart des Papiergeldes zeigt, welch ein immens hoher gestalterischer Aufwand betrieben wird, um dem Geld symbolhaft Werthaltigkeit und Identität zu verleihen (ausführlich Hartmann und Thiel 2016). Banknoten sind als „Visitenkarten des Staates“ immer auch

${ }^{12}$ Der Vorschlag der EU-Kommission vom 19.12.2016 (2016/0208 (COD)) bezieht sich dezidiert auf virtuelle Währungen, also digital repräsentierte Werte, die digital übertragen, gespeichert oder gehandelt werden können und von natürlichen oder juristischen Personen als Zahlungsmittel verwendet werden, jedoch weder gesetzliches Zahlungsmittel noch Giralgeld oder E-Geld im Sinne der zweiten Zahlungsdienstrichtlinie (PSD II) sind.

13 „Local currencies (also known as complementary currencies) that are used in very limited networks such as a city or a region and among a small number of users should not be considered as virtual currencies" (8) http://data.consilium.europa.eu/doc/document/ST-15605-2016-INIT/en/pdf [20.09.2017]. 
symbolischer Ausdruck eines bestimmten Staatsverständnisses, eines gesellschaftlichen Entwicklungsstands und einer nationalen Identität. Gleichermaßen müssen komplementäre Währungen ihre Inhalte symbolisch vermitteln - schließlich besteht ihre Daseinsberechtigung gerade darin, als „,besseres Geld“ bestimmte regionale, soziale oder ökologische Ziele zu verfolgen. Zudem soll das Komplementärgeld werthaltig und vertrauenswürdig wirken, sprich eine „aura of “moneyness"“ (CCIA 2015, S. 141) ausstrahlen. Ein neueres Handbuch für die Erstellung von Komplementärwährungen macht deutlich: „Cheaply printed or poorly designed notes and coins are less likely to be taken seriously by users, whose belief in the currency is central to its success" (CCIA 2015, S. 141). Dementsprechend sind viele der bisherigen Regionalgelder aufwendig gestaltet: Die 'moneyness' wird symbolisiert durch die Verwendung von geldtypischen materiellen und gestalterischen Elementen wie dem Format der Scheine, dem kompositorischen Aufbau, Sicherheitsmerkmalen, Signaturen, typischen Guillochen und Farbgebungen. Die Ziele des Komplementärgeldes spiegeln sich in der Bildgebung, bei der etwa lokale Bauwerke (regionaler Bezug), Landschaften (ökologischer Bezug) oder Werke örtlicher Künstler (ideeller Bezug) abgebildet werden. Die symbolische Gestaltung des Geldes dient so der Vermittlung bestimmter Intentionen, dem Aufbau einer territorial verorteten Gemeinschaft und der Konstitution einer gemeinschaftlichen Identität (siehe dazu Fare et al. 2015). Bei virtuellen Währungen ist dieses symbolische Gestaltungspotential nur eingeschränkt (bei Bankkarten) oder gar nicht (bei elektronischen Verrechnungssystemen) gegeben. Eine rein virtuelle Währung wird nur noch als abstraktes „Accounting“ wahrgenommen und nicht mehr als konkretes „Ding“. Giralgeld benötigt deswegen die gleichzeitige Existenz des Bargelds, das es als „symbolischen Anker“ gedanklich greifbar macht. Fällt dieser symbolische Anker der Dinglichkeit zunehmend weg, führt dies auch zu einem anderen Umgang mit Geld. Dieser Gedanke findet sich schon bei Georg Simmel. In seinem 1900 erschienenen Werk „Philosophie des Geldes“ schreibt Simmel, Geld werde mit zunehmender Bedeutung immer ,indifferenter, farbloser, allem einzelnen gegenüber objektiver“ (Simmel 1989, S. 266), bis es schließlich eine „völlig objektive, an sich qualitätslose Instanz“ (Simmel 1989, S. 80) bildet, die von allen spezifischen Inhalten gelöst ist und in reiner Quantität besteht. Analog dazu finde eine zunehmende „Entstofflichung“ des Geldes statt. Simmel zufolge ist in solchen unbaren, kreditbasierten Zahlungsweisen immer auch eine „Versuchung zum Leichtsinn“ (Simmel 1989: , S. 667) angelegt. Studien über Veränderungen des Zahlungsverhaltens nach dem Aufkommen der Konsum- und Hypothekenkredite (in den 1950ern) und der Kreditkarten (in 
den 1970ern) bestätigen seine These: Vor allem in den USA stieg in der Folge sowohl der Konsum als auch die Verschuldung (Ritzer 1995).

Dies führt uns zum Aspekt der Zahlungsgewohnheiten. Vielen Menschen ist bewusst, dass sie mit einem virtuellen, relativ abstrakten Zahlungsmittel mitunter mehr Geld ausgeben als mit einem Geldschein. Sie nutzen das Bargeld als Instrument ihrer Haushaltsplanung. Auch Gewohnheiten und historische Währungserfahrungen tragen dazu bei, dass immer noch viel mit Bargeld bezahlt wird. Komplementärwährungssysteme müssen sich - wollen sie eine gewisse Verbreitung erreichen - auf diese Zahlungsgewohnheiten einstellen. Laut einer Studie der Bundesbank wird in Deutschland am häufigsten bar bezahlt $-79,1 \%$ aller Kauftransaktionen werden mit Münzen und Scheinen beglichen (Deutsche Bundesbank 2015, S. 26) ${ }^{14}$. Jeder Dritte zahlt sogar ausschließlich bar, jeder zweite macht die Wahl des Zahlungsmittels von der mitgeführten Bargeldmenge abhängig (Deutsche Bundesbank 2015: 39). Auch die Höhe des zu zahlenden Betrags ist für viele (46 \%) wichtig - kleinere Beträge bis $20 €$ etwa werden fast immer bar bezahlt. Erst bei Einkäufen über $50 €$ sinkt der Barzahleranteil signifikant (auf 41,9\%), und wenn mehr als $100 €$ fällig sind, kommt vorzugsweise die Giro- und Kreditkarte zum Einsatz (nur noch 30,2 \% Barzahler bei über 100 $€$ und $24,1 \%$ bei über $500 €$ ) (Deutsche Bundesbank 2015, S. 31). Solch höhere Beträge sind bei Komplementärwährungen eher die Seltenheit, meist wird das Äquivalent von zehn bis zwanzig Euro pro Bezahlvorgang ausgegeben. Und solche Summen zahlt man eben meist (noch) in bar. Die Zahlen der Bundesbank decken sich mit den Erfahrungen, die die Regionalwährung „Chiemgauer“ gemacht hat: Generell zahlt bei dieser ca. ein Drittel der Teilnehmer nur in bar, ein weiteres Drittel ausschließlich mit Karte und der Rest verwendet beides. Allerdings wird der elektronische Chiemgauer (v.a. aus Bequemlichkeitsgründen) immer stärker nachgefragt. Dies zeigt: Zahlungsgewohnheiten können (und werden) sich ändern.

Insgesamt schwindet somit der „Gewohnheitsvorteil“ der bargeldbasierten Komplementärwährungen, denn es wird zunehmend als bequemer und moderner wahrgenommen, unbar zu bezahlen. Was bleibt, sind die im Vergleich zu elektronischen Währungen größeren Möglichkeiten in der symbolisch-gestalterischen Vermittlung bestimmter Botschaften.

14 Die Studie fokussierte dabei ausschließlich das Bezahlverhalten am Erfüllungsort (Point-of-Sale), nicht regelmäßig wiederkehrende Zahlungen (z.B. Miete, Telefonrechnungen), die meist unbar beglichen werden. Betrachtet man statt der Transaktionszahl den Umsatz, so führt das Bargeld mit 53,2 \%, gefolgt von der girocard mit 29,4 \% und der Kreditkarte mit 3,9 \% (Deutsche Bundesbank 2015, S. 27). 


\section{Schlussfolgerung und Ausblick}

Bargeld existiert nicht nur in Form der offiziellen nationalstaatlichen Banknoten, sondern auch in vielen privaten Geldsystemen. Die zumeist idealistisch motivierten Regionalwährungen sind in den letzten Jahrzehnten weltweit entstanden. Seit einigen Jahren ist ihre Entwicklung jedoch rückläufig. Viele der neuen Komplementärwährungssysteme wenden sich vom Bargeld ab und präferieren elektronische Verrechnungssysteme oder gar Kryptowährungen. Nicht wenige der neuen „Komplementärgeldmacher“, maßgeblich jene aus der Kryptowährungs- und FinTech-Szene, sind überzeugt, die bisherigen Probleme der Komplementärwährungen ließen sich mit den neuen Datenbanktechnologien und Handyapplikationen beheben ${ }^{15}$. Ob sich dieser Trend bei den Komplementärwährungen durchsetzen wird, lässt sich derzeit nicht beantworten. Allerdings bietet das Medium Bargeld auch gewisse Vorteile, gerade für komplementäre Währungssysteme.

Diese Vorteile resultieren aus der Materialität des Bargeldes, aus seiner physisch greifbaren Form. Erstens bietet diese ein größeres Symbolisierungspotential. Banknoten können mehr und besser als virtuelles Geld mit ihrer symbolischen Gestaltung Inhalte und Botschaften transportieren. Gerade für Komplementärwährungen ist das essentiell, schließlich geht es ihnen nicht um einfache und universelle Verwendbarkeit, sondern um idealistische Ziele. Die symbolische Aufladung des komplementären Geldes trägt zur Rechtfertigung des damit verbundenen höheren Aufwands und der Kosten bei. Man könnte dies auch ganz profan als Marketingmaßnahme im „Wettstreit der Währungen“ sehen, in dem sich komplementäre Währungen mit einer Vielzahl von (bargeldlosen) Zahlungsinstrumenten, Kundenbindungssystemen und Kryptowährungen messen müssen. Zweitens resultiert aus der Materialität und Symbolik des Geldes eine je eigene Handlungsrationalität. Dass das physisch greifbare Bargeld anders, dinglicher, weniger leichtfertig erfahren und behandelt wird, wurde schon angesprochen. Geldsoziologisch lässt sich dieser Gedanke noch weiterführen. Simmel (1989) hat eindrücklich gezeigt, welche Auswirkungen der indifferente, objektivierende und quantifizierende Charakter von Geld hat: Es wirkt als zentraler Motor der Individualisierung, indem es die Abhängigkeit einer Person von einigen wenigen, persönlich bekannten Personen (der Dorfgemeinschaft, dem Lehnsherr etc.) auflöst und durch partielle Abhängigkeiten gegenüber zahlreichen diffusen, anonymen und depersonalisierten Anderen (dem Markt) ersetzt. Die so erzeugte Vielzahl versachlichter, anonymer Beziehungen gewährt

\footnotetext{
15 Siehe dazu den Bericht von Leander Bindewald von der 4. Internationalen Forschungskonferenz zur monetären Vielfalt in Barcelona im Mai 2017 unter http://monneta.org/news/bericht-4-internationaleforschungskonferenz-zur-monetaeren-vielfalt-barcelona-mai-2017/ [20.09.2017].
} 
dem Einzelnen einerseits selbstbestimmte Handlungschancen und selbstreferentielle Identität, „befreit“ ihn gewissermaßen. Andererseits werden so traditionelle Gemeinschaften und ihre identitätsstiftenden Beziehungen zerstört, mit Folgen wie Entfremdung, Unsicherheit und Sinnverlust (Simmel 1989, S. 456). Komplementärwährungen hingegen sind zwar Geld, jedoch kein absolut objektives und qualitätsloses. Im Gegenteil, sie sind nur begrenzt verwendbar und symbolisch stark aufgeladen. Diese räumlichen und ideellen „Grenzziehungen“ (vgl. Schröder 2016) relativieren die geldimmanente Unpersönlichkeit, Abstraktheit und Rechenhaftigkeit. Sie setzen quasi einen anderen Rahmen und stimulieren damit andere Austausch- und Kommunikationsprozesse. Komplementärgeld ist so weniger indifferent gegenüber Werten, Zeitpunkten und Tauschpartnern, es ist weniger abstrakt und somit näher am Alltagsverständnis (Hörisch 2000, S. 72; Rapp 2000, S. 100). Dies gelingt physisch existierenden Komplementärwährungen weitaus besser als rein virtuellen. Der dritte Vorteil von bargeldbasierten Währungen ist ihre Unabhängigkeit von Technik und die daraus resultierende weitgehende Anonymität. Die meisten virtuellen Währungen (blockchainbasierte Kryptowährungen mal ausgenommen) erfordern eine Anmeldung in den entsprechenden Zahlungsnetzwerken. Sämtliche getätigte Transaktionen werden dadurch gespeichert, was nicht unproblematisch ist, da nicht absehbar ist, wie welche Daten von wem genutzt werden. Hinzu kommt der Aspekt der Sicherheit, da kein technisches System unangreifbar ist (zu den Aspekten von Überwachung und Sicherheit siehe etwa Goodman 2016; Häring 2016).

Abschließend lässt sich damit festhalten: Die Zukunft des „,idealistischen Geldes“, der Komplementärwährungen, mag ungewiss sein. Doch vielleicht liegt sie gerade in dem scheinbar antiquierten Geldmedium „Bargeld“. 


\section{Literatur}

Becker, H. S. (1973): Außenseiter. Zur Soziologie abweichenden Verhaltens. Frankfurt a. M.: S. Fischer.

Blanc, J. (2011): Classifying "CCs": Community, complementary and local currencies' types and generations. International Journal of Community Currency Research 15 (2011), D 4-10.

Blanc, J.; Fare, M. (2013): Understanding the Role of Governments and Administrations in the Implementation of Community and Complementary Currencies. Annals of Public and Cooperative Economics, Vol. 84, Issue 1, S. 63-81.

Carruthers, B.; Espeland, W. (2002): Money, Meaning and Morality. In: N.W. Biggart (Hrsg.): Readings in economic sociology (S. 292-314). Malden, Mass.: Blackwell

CCIA (Community Currencies in Action) (2015): People Powered Money-Designing, developing \& delivering community currencies. http://b.3cdn.net/nefoundation/0dba46d13aa81f0fe3_zhm62ipns.pdf. Zugegriffen: 13.09.2017.

Degens, P. (2013): Alternative Geldkonzepte - ein Literaturbericht. MPIfG Discussion Paper 13/1. http://www.mpifg.de/pu/mpifg_dp/dp13-1.pdf. Zugegriffen: 25.09.2017

Deutsche Bundesbank (2015): Zahlungsverhalten in Deutschland 2014. Dritte Studie über die Verwendung von Bargeld und unbaren Zahlungsinstrumenten.

https://www.bundesbank.de/Redaktion/DE/Downloads/Veroeffentlichungen/Studien/zahlungsverha lten in deutschland 2014.pdf? blob=publicationFile. Zugegriffen: 25.09.2017.

European Central Bank (2012): Virtual Currency Schemes. https://www.ecb.europa.eu/pub/pdf/other/virtualcurrencyschemes201210en.pdf. Zugegriffen: 25.09.2017.

Fare, M.; Ahmed, P. (2017): Complementary Currency Systems and their Ability to Support Economic and Social Changes. Development and Change. doi:10.1111/dech.12322

Fare, M.; de Freitas, C.; Meyer, C. (2015): Territorial Development and Community Currencies: Symbolic Meanings in Brazilian Community Development Banks. International Journal of Community Currency Research, 19 (D), S. 6-17.

Ferreira, J.; Perry, M.; Subramanian, S. (2015): Spending Time with Money: From Shared Values to Social Connectivity. CSCW '15: Proceedings of the 18th ACM Conference on Computer Supported Cooperative Work \& Social Computing. doi:10.1145/2675133.2675230

Gabriel, G. (2002): Ästhetik und Rhetorik des Geldes. Stuttgart: Frommann-Holzboog.

Goodman, M. (2016): Future Crimes. Inside the Digital Underground and the Battle for our Connected World. New York: Anchor Books.

Gründler, E. (2005): Komplementäres Geld. Vorteile, Erscheinungsformen und Funktionsweisen. Zürich: MoneyMuseum.

Hardraht, K.; Godschalk, H. (2004): Komplementärwährungsgutachten. Erstellt im Auftrag der Sparkasse Delitzsch-Eilenburg. 
Häring, N. (2016): Die Abschaffung des Bargelds und die Folgen. Der Weg in die totale Kontrolle. Köln: Bastei Lübbe.

Hartmann, S.; Thiel, C. (Hrsg.) (2016): Der schöne Schein. Symbolik und Ästhetik von Banknoten. Regenstauf: Gietl.

Hoeben, C. (2003): Let's be a community. Community in local exchange trading systems. Groningen: Rijksuniversiteit.

Hörisch, J. (2000): Geld, Geist und Information. Kunstforum International, Bd. 149, S. $72-73$.

Jacob, J.; Brinkerhoff, M.; Jovic, E.; Wheatley, G. (2004): HOUR Town - Paul Glover and the Genesis and Evolution of Ithaca HOURS. International Journal of Community Currency Research 8, S. 29-39.

Jacobs, J.; Merlin, B.; Emiliy, J.; Wheatley, G. (2004): The Social and Cultural Capital of Community Currency. An Ithaca HOURS Case Study Survey. International Journal of Community Currency Research 8, S. 42-56.

Kennedy, M. (2005): Komplementärwährungen zur wirtschaftlichen Lösung sozialer Probleme. Zeitschrift für Sozialökonomie, 42, 144, S. 20-28.

Kennedy, M.; Lietaer, B. (2004): Regionalwährungen: Neue Wege zu nachhaltigem Wohlstand. München: Rieman.

Kirschner, A. (2011): The Burlington Currency Project: A History. International Journal of Community Currency Research 15 (A), S. 42-55.

Kleine, J.; Krautbauer, M.; Weller, T. (2013): Cost of Cash: Status Quo und Entwicklungsperspektiven in Deutschland. Steinbeis Research Center for Financial Services, München. http://www.steinbeisresearch.de/images/pdf-documents/CFP_Cost_Of_Cash_Studie_Steinbeis_Deutsch.pdf. Zugegriffen: 20.09.2017.

Lietaer, B.; Ulanowicz, R.; Goerner, S. (2008): Wege zur Bewältigung systemischer Bankenkrisen. http://www.lietaer.com/images/White_Paper_Lietaer_Deutsch.pdf. Zugegriffen: 30.03.2009.

Meier, D. (2001): Tauschringe als besondere Bewertungssysteme in der Schattenwirtschaft: Eine theoretische und empirische Analyse. Berlin: Ducker\&Humblot.

North, P. (2007): Money and Liberation: The Micropolitics of the Alternative Currency Movement. Minneapolis: University of Minnesota Press.

North, P. (2010): Local Money. Totnes: Transition Books.

Plettenbacher, T. (2008): Neues Geld. Neue Welt. Die drohende Wirtschaftskrise - Ursachen und Auswege. Wien: Planet Verlag.

Rapp, J. (2000): Sozialmaschine Geld. Kunstforum International 149, S. 100-102.

Reifner, U. (2017): Das Geld. Band 3. Recht des Geldes. Regulierung und Gerechtigkeit. Wiesbaden: Springer Fachmedien.

Ritzer, G. (1995): Expressing America - A Critique of the Global Credit Card Society. Tousand Oaks/ London/ New Delhi: Pine Forge Press. 
Rogoff, K. (2016): Der Fluch des Geldes: Warum unser Bargeld verschwinden wird. München: FinanzBuch Verlag.

Sademach, M. (2010): Regionalwährungen in Deutschland und ihre Münzen. Moneytrend 11/2010, S. 170-173.

Schröder, R. (2016): The double-edgedness of monetary multiplicity: How can complementary currencies promote the case for social justice? Vortrag auf der Polanyi International Seminar Societies in transition: Social and solidarity economy, the commons, public action and livelihood CNAM, Paris (France) $\cdot 19-20$ May 2016.

Schroeder, R. (2015): The Financing of Complementary Currencies: Problems and Perspectives. International Journal of Community Currency Research 19, D106-113.

Seyfang, G.; Longhurst, N. (2012): Money, Money, Money? A scoping study of grassroots complementary currencies for sustainability. https://grassrootsinnovations. files.wordpress.com/2012/05/seyfang-and-longhurst-2012-moneymoney-money.pdf. Zugegriffen: 28.08.17.

Seyfang, G.; Longhurst, N. (2013): Growing green money? Mapping community currencies for sustainable development. Ecological Economics 86, S. 65-77.

Seyfang, G. (2002): Tackling social exclusion with community currencies: learning from LETS to Time Banks. International Journal of Community Currency Research 6.

Seyfang, G.; Pearson, R. (2000): Time for Change: International experience in community currencies. Development 43, 4, S. 56-60.

Simmel, G. (1989 [1900]): Philosophie des Geldes. Frankfurt a. M.: Suhrkamp.

Thiel, C. (2011): Das »bessere« Geld. Eine ethnographische Studie über Regionalwährungen. Wiesbaden: VS Verlag.

Thiel, C. (2013): Der schöne Schein. Banknoten als Untersuchungsgegenstand einer visuellen Soziologie. Soziale Welt 1-2, S. 191-216.

Thiele, C. (2015): Diskussion um das Bargeld: Hätte eine Abschaffung von Banknoten und Münzen wirklich Vorteile? Ifo Schnelldienst 13, S. 3-6.

Zelizer, V. (1989): The social meaning of monies: »Special monies«. American Journal of Sociology 95, S. 342-377. 